\title{
A CRITERION FOR THE NORMALITY OF UNBOUNDED OPERATORS AND APPLICATIONS TO SELF-ADJOINTNESS
}

\author{
MOHAMMED HICHEM MORTAD
}

\begin{abstract}
In this paper we give and prove a criterion for the normality of unbounded closed operators, which is a sort of a maximality result which will be called "double maximality". As applications, we show, under some assumptions, that the sum of two symmetric operators is essentially self-adjoint; and that the sum of two unbounded normal operators is essentially normal. Some other important results are also established.
\end{abstract}

\section{INTRODUCTION}

First, we assume the reader is familiar with notions and results about bounded and unbounded linear operators. Some general references are [2, 5, 6, 12, 14, 16, 19,.

Let us, however, recall the next known result which will be useful.

Lemma 1 ([19]). If $A$ and $B$ are densely defined and $A$ is invertible with inverse $A^{-1}$ in $B(H)$, then $(B A)^{*}=A^{*} B^{*}$.

In order to avoid an eventual confusion for Banach algebraists, we say here that an unbounded densely defined closeable operator $A$ is essentially normal if its closure $\bar{A}$ is normal.

It is known that self-adjoint operators are maximally symmetric. Hence, any closed operator $A$ such that $A A^{*} \subset A^{*} A$ is automatically normal for $A A^{*}$ and $A^{*} A$ are both self-adjoint. It is also well-known that normal operators are maximally normal. For some other maximality results, see [3] and [11].

However, there are some situations which one encounters sometimes and which are not covered by any of the results cited above. For instance what can we say about a closed or non closed densely defined operator $S$ obeying $T \subset S S^{*}$ and $T \subset S^{*} S$ (where $T$ is just densely defined)? The answer will be given in Theorem 2 below. Then we prove a more general result. It mainly gives conditions on when $T \subset R$ and $T \subset S$ imply that $S=R$ ? See Theorem 3 ,

As an important consequence of Theorem 2 we establish a new result on the essential self-adjointness of the sum of two unbounded symmetric operators (see Theorem (4).

Then we prove a result on the normality of the sum of two unbounded normal operators (cf [9]). A certain form of commutativity (not strong commutativity though) is required.

For the reader's convenience, let us gather in one theorem some of the famous results on the self-adjointness of the sum of two self-adjoint:

2010 Mathematics Subject Classification. Primary 47A05; Secondary 47B25.

Key words and phrases. Unbounded operators. Self-adjoint, closed and normal operators. Operator sums.

Partially supported by "Laboratoire d'Analyse Mathématique et Applications". 
Theorem 1. Let $A$ and $B$ be two operators with domains $D(A)$ and $D(B)$ respectively, such that $A+B$ is densely defined 1 . Then $A+B$ is:

(1) self-adjoint on $D(A)$ if $A$ and $B$ are self-adjoint, and if $B$ is bounded.

(2) self-adjoint on $D(A) \cap D(B)$ whenever $A$ and $B$ are commuting self-adjoint and positive operators (see [12]).

(3) self-adjoint on $D(A) \cap D(B)$ whenever $A$ and $B$ are anticommuting selfadjoint operators (see [18]).

(4) (Kato-Rellich) self-adjoint on $D(A)$ if $B$ is symmetric and $A$-bounded with relative bound $a<1$, and $A$ is self-adjoint (see [13]).

For papers treating similar questions on sums, see [8, 9, 18].

\section{Main Results}

The first important result of the paper is a "double maximality" one:

Theorem 2. Let $S, T$ be two densely defined unbounded operators on a Hilbert space $H$ with respective domains $D(S)$ and $D(T)$. Assume that

$$
\left\{\begin{array}{l}
T \subset S^{*} S \\
T \subset S S^{*}
\end{array}\right.
$$

Let $D \subset D(T)\left(\subset D\left(S^{*} S\right) \cap D\left(S S^{*}\right)\right)$ be dense.

(1) Assume that $D$ is a core, for example, for $S^{*} S$. If $S$ is closed, then $S$ is normal.

(2) If $S$ is not closed, then $\bar{S}$ is normal if $D$ is a core for $S^{*} \bar{S}$.

Proof.

(1) Recall that $S S^{*}$ and $S^{*} S$ are both self-adjoint on the respective domain $D\left(S S^{*}\right)$ and $D\left(S^{*} S\right)$. We clearly have

$$
0_{D(T)}=T-T \subset S^{*} S-S S^{*} .
$$

Hence

$$
S^{*} S-S S^{*} \subset\left(S^{*} S-S S^{*}\right)^{*} \subset\left[0_{D(T)}\right]^{*}=0_{H}
$$

If we denote the restriction of $S^{*} S$ to $D$ by $S^{*} S_{D}$, then

$$
S^{*} S_{D} \subset S^{*} S-S S^{*}+S S^{*} \subset S S^{*} \text {. }
$$

Since $D$ is a core for $S^{*} S$, we have

$$
S^{*} S=\overline{S^{*} S_{D}}
$$

Since self-adjoint operators are maximally symmetric, passing to closures in the inclusions (11) we see that we have

$$
S^{*} S=S S^{*}
$$

proving the normality of $S$.

(2) If $S$ is not closed, the proof is very similar. We just observe that $S^{*}$ is densely defined and that in this case

$$
S^{*} \bar{S}-\bar{S} S^{*} \subset\left(S^{*} S-S S^{*}\right)^{*} \subset\left[0_{D(T)}\right]^{*}=0_{H} .
$$

\footnotetext{
${ }^{1}$ Kosaki [7] gave explicit examples of unbounded densely defined self-adjoint positive operators $A$ and $B$ such that $D(A) \cap D(B)=\{0\}$.
} 
Remark. Of course, if we only have

$$
\left\{\begin{array}{l}
T \subset S^{*} S \\
T \subset S S^{*}
\end{array}\right.
$$

(i.e. without any "core condition"), then $S$ is not necessarily normal even if it is closed as seen in the following example:

Example. Let $S$ be defined by $S f(x)=-f^{\prime}(x)$ on $D(S)=\left\{f \in L^{2}(0,1): f^{\prime} \in\right.$ $\left.L^{2}(0,1)\right\}$. Then $S$ is densely defined and closed, but it is not normal. Indeed,

$$
S^{*} f(x)=-f^{\prime}(x) \text { on } D\left(S^{*}\right)=\left\{f \in L^{2}(0,1): f^{\prime} \in L^{2}(0,1), f(0)=f(1)=0\right\}
$$

so that

$$
S S^{*} f(x)=S^{*} S f(x)=-f^{\prime \prime}(x)
$$

with

$$
D\left(S S^{*}\right)=\left\{f \in L^{2}(0,1): f^{\prime \prime} \in L^{2}(0,1), f(0)=f(1)=0\right\}
$$

and

$$
D\left(S^{*} S\right)=\left\{f \in L^{2}(0,1): f^{\prime \prime} \in L^{2}(0,1), f^{\prime}(0)=f^{\prime}(1)=0\right\} .
$$

Hence $S$ is not normal. Let $T$ be defined by $T f(x)=-f^{\prime \prime}(x)$ on

$$
D(T)=\left\{f \in L^{2}(0,1): f^{\prime \prime} \in L^{2}(0,1), f(0)=f(1)=0=f^{\prime}(0)=f^{\prime}(1)\right\} .
$$

Then

$$
\left\{\begin{array}{l}
T \subset S^{*} S, \\
T \subset S S^{*},
\end{array}\right.
$$

$T$ and $S$ are densely defined, $S$ is closed, and yet as we have just seen $S$ is not normal.

Adopting the same proof as that of Theorem 2 we can easily generalize the latter theorem to the following "double maximality" result which is, by the way, stronger than Theorem 5.31 in [19] (in [19, the coming $T$ was assumed to be symmetric and it was also assumed that $D(R) \subset D(S)$ ). We leave the proof to the reader.

Theorem 3. Let $R, S, T$ be three densely defined unbounded operators on a Hilbert space $H$ with respective domains $D(R), D(S)$ and $D(T)$. Assume that

$$
\left\{\begin{array}{l}
T \subset R, \\
T \subset S .
\end{array}\right.
$$

Assume further that $R$ and $S$ are self-adjoint. Let $D \subset D(T)(\subset D(R) \cap D(S))$ be dense. Let $D$ be a core, for instance, for $S$. Then $R=S$.

Remark. After I proved Theorem 2, I came across a result due to Stochel-Szafraniec [17] which I was not aware of. The authors proved a similar result to mine. They further assumed that $S^{*} S_{D}$ was essentially self-adjoint (something obtained in my proof) but then they obtained in their proof that $D$ is a core for $S^{*} S$ (something assumed in my theorem). In fact, it is well-known that a linear subspace $D$ is core for a self-adjoint operator $A$ iff $A$ restricted to $D$ is essentially self-adjoint.

Now we prove an interesting result on the sum of two symmetric operators. This is the natural generalization of the known result on the sum of two bounded symmetric (or self-adjoint) operators. 
Theorem 4. Let $A$ and $B$ be two unbounded symmetric operators. Let $D$ be a dense linear manifold contained in the domain $D\left[(A+B)^{2}\right]$. If $D$ is a core for $(A+B)^{*}(\overline{A+B})$, then $A+B$ is essentially self-adjoint on $D$.

Remark. The hypothesis $A$ and $B$ being symmetric is weak but the assumption $(A+B)^{2}$ being densely defined is strong (where $A+B$ is symmetric).

Remember that Chernoff [1] (see also [4, 10, 15]) gave an explicit example of a symmetric, closed and semi-bounded operator $A$ satisfying $D\left(A^{2}\right)=\{0\}$ (hence $D\left(A^{2}\right)$ is far from being dense!).

For further investigations on when $D\left(A^{n}\right)$ is dense, see the intensive work [15].

Remark. In practise, especially when dealing with partial differential operators, it is usually fairly simple to find the appropriate dense domain $D$. In this case, the domain par excellence is $C_{0}^{\infty}\left(\mathbb{R}^{n}\right)$.

We may exploit Chernoff's counterexample to show the importance of the dense manifold $D$ in the foregoing theorem as seen in

Example. Let $A$ be a closed and symmetric operator such that $D\left(A^{2}\right)=\{0\}$. Hence $A$ is not self-adjoint (if it were, then $A^{2}$ would be self-adjoint too and $D\left(A^{2}\right)$ would then be dense). Set $B=A$, then

$$
D\left[(A+B)^{2}\right]=D\left(4 A^{2}\right)=D\left(A^{2}\right)=\{0\} .
$$

Hence $D\left[(A+B)^{2}\right]$ does not contain any dense set $D$. Finally, as observed above,

$$
\overline{A+B}=\overline{A+A}=2 A
$$

is not self-adjoint.

Now we prove Theorem 4

Proof. Since $D \subset D\left[(A+B)^{2}\right],(A+B)^{2}$ is densely defined, and then so is $A+B$. Hence $A+B$ is closeable for

$$
A+B \subset A^{*}+B^{*} \subset(A+B)^{*} .
$$

Then we have

$$
A+B \subset(A+B)^{*}=(\overline{A+B})^{*} \Longrightarrow \overline{A+B} \subset(\overline{A+B})^{*},
$$

that is $\overline{A+B}$ is symmetric. We also have

$$
A+B \subset(A+B)^{*} \Longrightarrow(A+B)^{2} \subset(A+B)^{*}(A+B) .
$$

Similarly,

$$
(A+B)^{2} \subset(A+B)(A+B)^{*}
$$

Hence Theorem 2 implies that $\overline{A+B}$ is normal. Since symmetric normal operators are self-adjoint, we immediately deduce that $A+B$ is essentially self-adjoint on $D$.

We have the analog of Theorem 4 for a finite family of operators. The proof is left to the interested reader.

Theorem 5. Let $\left(A_{k}\right)_{1 \leq k \leq n}$ be a family of symmetric operators. Let $D$ be a dense linear manifold contained in the domain $D\left[\left(A_{1}+\cdots+A_{n}\right)^{2}\right]$. If $D$ is a core for $\left(A_{1}+\cdots+A_{n}\right)^{*}\left(\overline{A_{1}+\cdots+A_{n}}\right)$, then $A_{1}+\cdots+A_{n}$ is essentially self-adjoint on $D$. 
It is a classic matter to take an unbounded normal operator $A$ and then set $B=-A$ to see that $A+B$ is not normal (as it is not closed). But in this case $A+B$ is essentially normal for its closure is worth "0" defined everywhere. This observation has inspired me to give and prove the following result:

Proposition 1. Let $A$ and $B$ be two unbounded normal operators obeying $A B^{*}=$ $B^{*} A$. Assume that only $B$ is invertible. Let $D$ be a dense linear manifold contained in the domains of $A^{*} A, B^{*} A, A^{*} B$ and $B^{*} B$. Then $A+B$ is essentially normal on $D$ if $D$ is a core for $(A+B)^{*}(A+B)$.

Proof. We have

$$
\begin{aligned}
(A+B)(A+B)^{*} & \supset(A+B)\left(A^{*}+B^{*}\right) \\
& =A\left(A^{*}+B^{*}\right)+B\left(A^{*}+B^{*}\right) \\
& \supset A A^{*}+A B^{*}+B A^{*}+B B^{*} .
\end{aligned}
$$

Similarly, we obtain

$$
(A+B)^{*}(A+B) \supset A^{*} A+B^{*} A+A^{*} B+B^{*} B .
$$

Since $A B^{*}=B^{*} A$ and $B$ is invertible, Lemma 1 yields $A^{*} B \subset B A^{*}$. Hence

$$
A^{*} A+B^{*} A+A^{*} B+B^{*} B \subset A A^{*}+A B^{*}+B A^{*}+B B^{*} \text {. }
$$

Set $Q=A^{*} A+B^{*} A+A^{*} B+B^{*} B$. Then

$$
(A+B)(A+B)^{*} \supset Q \text { and }(A+B)^{*}(A+B) \supset Q .
$$

Since $Q$ is densely defined, Theorem 2 yields the desired result.

The last result of the paper concerns the normality of the sum too. It is wellknown that the sum of two bounded commuting normal operators is normal. This was generalized to the case where one operator is unbounded (see 9]). It is also known that the sum of two strongly anti-commuting unbounded self-adjoint operators is self-adjoint (see [18]). This result, however, cannot be generalized to the case of two strongly anticommuting normal operators even for the case of two bounded normal operators. Indeed, let

$$
A=\left(\begin{array}{cc}
2 & 0 \\
0 & -2
\end{array}\right) \text { and } B=\left(\begin{array}{cc}
0 & 1 \\
-1 & 0
\end{array}\right) \text {. }
$$

Then $A$ and $B$ are both normal. They anticommute because

$$
A B=-B A=\left(\begin{array}{ll}
0 & 2 \\
2 & 0
\end{array}\right)
$$

but as one can easily check

$$
A+B=\left(\begin{array}{cc}
2 & 1 \\
-1 & -2
\end{array}\right)
$$

is not normal.

Nevertheless, we have the following result:

Theorem 6. Let $A$ be an unbounded normal operator and let $B$ be bounded and self-adjoint. If $B A^{*} \subset-A B$, then $A+B$ is normal.

The proof requires the following lemma whose proof is very akin to the one in [9]. 
Lemma 2. Let $A$ be an unbounded normal operator with domain $D(A)$. Let $B$ be a bounded self-adjoint operator defined on a Hilbert space $\mathcal{H}$. If $B A^{*} \subset-A B$, then

$$
D\left[A^{*}(A+B)\right]=D\left(A^{*} A\right) \text { and } D\left[A\left(A^{*}+B\right)\right]=D\left(A A^{*}\right),
$$

so that

$$
A^{*}(A+B)=A^{*} A+A^{*} B \text { and } A\left(A^{*}+B\right)=A A^{*}+A B .
$$

Now we prove Theorem 7

Proof. Since $B$ is bounded (and self-adjoint), we have

$$
A+B \text { is closed and }(A+B)^{*}=A^{*}+B^{*}\left(=A^{*}+B\right) .
$$

Now thanks to Lemma 2 we have

$$
(A+B)^{*}(A+B)=A^{*} A+A^{*} B+B A+B^{2} .
$$

and

$$
(A+B)(A+B)^{*}=A A^{*}+A B+B A^{*}+B^{2} .
$$

Since $B A^{*} \subset-A B$, we have $B A \subset-A^{*} B$ (by the Fuglede-Putnam theorem), so that

$$
B A^{*}+A B \subset 0 \text { and } B A+A^{*} B \subset 0 .
$$

Hence

$$
(A+B)(A+B)^{*}=A A^{*}+A B+B A^{*}+B B^{2} \subset A A^{*}+B^{2}
$$

and

$$
(A+B)^{*}(A+B)=A^{*} A+A^{*} B+B A+B^{2} \subset A^{*} A+B^{2} .
$$

Since $A A^{*}$ is self-adjoint and $B^{2}$ is self-adjoint (and bounded), $A A^{*}+B^{2}$ is selfadjoint too. Since $A+B$ is closed, $(A+B)(A+B)^{*}$ is self-adjoint. But, self-adjoint operators are maximally symmetric. Thus

$$
(A+B)(A+B)^{*}=A A^{*}+B^{2} .
$$

Similarly, we obtain

$$
(A+B)^{*}(A+B)=A^{*} A+B^{2} .
$$

Therefore, and by the normality of both $A$ and $B$, we get that

$$
(A+B)(A+B)^{*}=(A+B)(A+B)^{*},
$$

completing the proof.

Very similarly, we also have

Theorem 7. Let $A$ and $B$ be two normal operators such that only $B$ is bounded. If $B A^{*} \subset-A B^{*}$ and $B^{*} A \subset-A^{*} B$, then $A+B$ is normal. 


\section{REFERENCES}

[1] P. R. Chernoff, A Semibounded Closed Symmetric Operator Whose Square Has Trivial Domain, Proc. Amer. Math. Soc., 89/2 (1983) 289-290.

[2] J. B. Conway, A Course in Functional Analysis, Springer, GTM 68 (1990), 2nd edition.

[3] A. Devinatz, A. E. Nussbaum, J. von Neumann, On the Permutability of Self-adjoint Operators, Ann. of Math. (2), 62 (1955), 199-203.

[4] J. Dixmier, L'adjoint du Produit de Deux Opérateurs Fermés, Annales de la faculté des sciences de Toulouse 4ème Série, 11 (1947) 101-106.

[5] I. Gohberg, S. Goldberg, M. A. Kaashoek, Basic Classes of Linear Operators, Birkhäuser Verlag, Basel, 2003.

[6] T. Kato, Perturbation Theory for Linear Operators, 2nd Edition, Springer, 1980.

[7] H. Kosaki, On Intersections of Domains of Unbounded Positive Operators, Kyushu J. Math., 60/1 (2006) 3-25.

[8] M. H. Mortad, On the Adjoint and the Closure of the Sum of Two Unbounded Operators, Canad. Math. Bull., 54/3 (2011) 498-505. DOI:10.4153/CMB-2011-041-7.

[9] M. H. Mortad, On the Normality of the Sum of Two Normal Operators, Complex Anal. Oper. Theory, 6/1 (2012), 105-112. DOI: 10.1007/s11785-010-0072-7.

[10] M. Naimark, On the Square of a Closed Symmetric Operator, Dokl. Akad. Nauk SSSR, 26 (1940) 866-870; ibid. 28 (1940), 207-208.

[11] A. E. Nussbaum, A Commutativity Theorem for Unbounded Operators in Hilbert Space, Trans. Amer. Math. Soc. 140 (1969), 485-491.

[12] C. Putnam, Commutation Properties of Hilbert Space Operators, Springer, 1967.

[13] M. Reed, B. Simon, Methods of Modern Mathematical Physics, Vol. 2: Fourier Analysis, Self-Adjointness, Academic Press, 1975.

[14] W. Rudin, Functional Analysis, McGraw-Hill, 1991 (2nd edition).

[15] K. Schmüdgen, On Domains of Powers of Closed Symmetric Operators, J. Operator Theory, 9/1 (1983) 53-75.

[16] K. Schmüdgen, Unbounded Self-adjoint Operators on Hilbert Space, Springer GTM 265 (2012).

[17] J. Stochel, F. H. Szafraniec, Domination of unbounded operators and commutativity, J. Math. Soc. Japan 55/2 (2003), 405-437.

[18] F. H. Vasilescu, Anticommuting Selfadjoint Operators, Rev. Roumaine Math. Pures Appl. 28/1 (1983), 76-91.

[19] J. Weidmann, Linear operators in Hilbert spaces (translated from the German by J. Szücs), Srpinger-Verlag, GTM 68 (1980).

Department of Mathematics, University of Oran, B.P. 1524, El Menouar, Oran 31000. Algeria.

Mailing address:

Dr Mohammed Hichem Mortad

BP 7085 Es-SEDDIKIA

ORAN

31013

Algeria

E-mail address: mhmortad@gmail.com, mortad@univ-oran.dz. 